\title{
Examining Serotonin Function: A Modified Technique for Rapid Tryptophan Depletion
}

Lois E. Krahn, M.D., Peter Y. Lu, M.D., George Klee, M.D., Ph.D., Pedro R. Delgado, M.D., Slong-Chi Lin, M.D., and Ralf C. Zimmermann, M.D.

Tryptophan (TRP) depletion was used to study serotonin because the ratio of TRP to large neutral amino acids (TRP/ $L N A A)$ determines the quantity of TRP that enters the brain. Because TRP is not universally available, a modified technique of TRP depletion was developed where a $1 / 4$ strength preparation of an amino acid mixture (AAM) replaces TRP as the placebo. Seven healthy subjects could not differentiate between the preparations in this double-blind, placebo-controlled study. Urinary 6-hydroxymelatonin sulfate (6-MS) was monitored as a biochemical marker of serotonin. The TRP/LNAA ratio ( $G G=0.0001)$ and $6-M S$ secretion $(G G=0.024)$ were decreased, but placebo TRP levels ( $G G=0.062)$ were not altered significantly. This modified technique facilitates the sue of TRP depletion in clinical research.

[Neuropsychopharmacology 15:325-328, 1996]
KEY WORDS: Mood; Tryptophan; Melatonin; Competing amino acids; Serotonin

In recent years rapid tryptophan (TRP) depletion using a specified amino acid mixture (AAM) has been used to study serotonin in psychiatric disorders, including depression (Delgado et al. 1990, 1994), obsessive-compulsive disorder (Lucca et al. 1992), bulimia (Weltzin et al. 1994), and schizophrenia (Rosse et al. 1992). The widespread use of this valuable research tool has been limited in double-blind placebo-controlled studies because the placebo drink consisted of AAM and TRP. When TRP was associated with eosinophilia-myalgia syndrome, the FDA removed TRP from the U.S. market in 1989 (CDC 1991). An alternative technique has been developed and used in several studies (Moreno et al.

From the Department of Psychiatry (LEK), the Division of Reproductive Endocrinology (PYL), the Division of Metabolic and Hematologic Biochemistry (GK), Mayo Clinic and Foundation, Rochester MN; the Department of Psychiatry (PRD), University of Arizona, Tucson AZ; the Department of Psychiatry (S-CL), Mayo Clinic and Foundation, Jacksonville FL; and the Section of Assisted Reproduction (RCZ), Columbia University, New York.

Address correspondence to: Lois E. Krahn, M.D., Department of Psychiatry and Psychology, Mayo Clinic and Foundation, Rochester, MN 55905 .

Received July 27, 1995; revised November 9, 1995; accepted December 7, 1995
1995). This study aims to develop a control drink without TRP with an impact on brain TRP levels similar to the original control drink.

\section{MATERIAL AND METHODS}

Eight healthy subjects ( 4 men, 4 women) were recruited by a classified advertisement. Their age was $25.6 \pm 2.8$ years (mean $\pm \mathrm{SE}$ ). No subject had a history of past psychiatric or medical disorders. Participants were not taking any medications other than one woman taking acyclovir, which does not interfere with indoleamine function. Screening included a semistructured psychiatric interview, physical examination, and laboratory tests. The women had regular menstrual cycles ( 26 to 32 days in duration) and were studied in the follicular phase (days 3-10). This study was approved by the Institutional Review Board of the Mayo Clinic and Foundation, and all participants provided written informed consent.

The active and control conditions were separated by 4 weeks in this double-blind, placebo-controlled study. The procedure was the same as described previously, apart for the fact that the control drink was $1 / 4$ strength of the active and contained no TRP (Zimmermann et al. 
1993). Patients consumed a low-TRP meal (160 mg TRP/day) at 12:00 and 18:00 on day 1 and at 8:00 on day 2. Other than the amino acid drink at 15:00 on day 2, subjects did not eat until 8:00 on day 3 . The drinks were prepared by pharmacy staff, and the investigators were not present at the time the AAM was administered. Subjects were asked during subsequent ratings whether they thought they had received the active or control preparation. Blood was drawn at the following time points: 14:00 (baseline), 17:00, 19:00, 21:00, 23:00, 01:00, and 06:00. Urine was collected at 8-hour intervals starting at 07:00. TRP values were determined with highperformance liquid chromatography and 6-MS with a radioimmunoassay kit (Zimmermann et al. 1993).

Mean total TRP, large neutral amino acids (LNAA), TRP/LNAA ratio, and 6-hydroxymelatonin (6-MS) values were calculated by averaging the values from the serum or urine collected for each time point for all subjects for each condition. A two-way analysis of variance (ANOVA) was used to evaluate the interaction between test day (active versus control) and control using the Greenhouse-Geisser correction procedure and post hoc paired $t$ tests. A one-way ANOVA was used with degrees of freedom with the Greenhouse-Geisser correction procedure and post hoc paired $t$ tests when appropriate to analyze changes over time compared to baseline within each condition for TRP, LNAA, and the TRP/ LNAA ratio.

\section{RESULTS}

When questioned by investigators, the subjects could not distinguish between the preparations. Subjects tolerated the protocol with no side effects other than mild to moderate nausea developing approximately $60 \mathrm{~min}$ utes after both the control and active drinks. Nausea subsided after several hours. One participant, a 39-yearold woman, dropped out of the study after the first visit because of the moderately severe nausea she experienced after the full-strength drink. This subject was not included because it was not possible to interpret the data.

A two-way ANOVA revealed a significant difference in total plasma TRP levels between the full and 1/4 strength conditions $(F=6.2, \mathrm{df}=6,36, \mathrm{GG}=0.016)$ at all time points except 14:00 and 17:00 (Figure 1). Total plasma TRP levels decreased significantly within the active condition $(F=12.3, \mathrm{df}=6,36, \mathrm{GG}=0.004)$, with all time points being significantly below baseline. During the control condition, TRP changed significantly $(F=7.47, \mathrm{df}=6,36, \mathrm{GG}=0.004)$ with the 17:00 below and 06:00 values above the baseline value.

A two-way ANOVA revealed a significant difference between the full and $1 / 4$ strength preparation for LNAA ( $F=39.1$, df $=6,36$, GG $=0.0001$ ) for all time

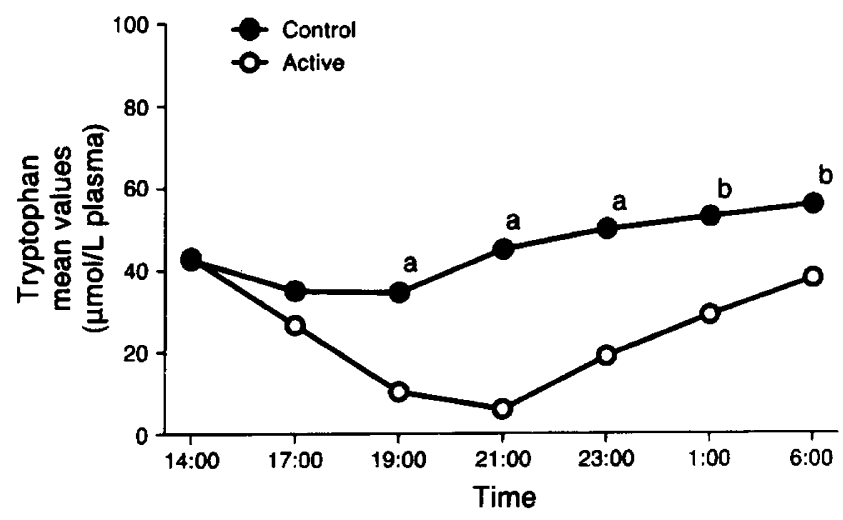

Figure 1. Mean total tryptophan values. Significant difference between active and control $(n=7), a, p<.005 ; b, p<.02$.

points except 14:00 (Figure 2). The change in LNAA was significant at all time points $(F=64.7, \mathrm{df}=6,36$, $\mathrm{GG}=0.0001$ ) for all time points except 14:00 (Figure 2). The change in LNAA was significant at all time points $(F=64.7, \mathrm{df}=6,36, \mathrm{GG}=0.0001)$ only with the active condition.

A two-way ANOVA for the TRP/LNAA ratio showed a significant difference between the two conditions $(F=$ $10.87, \mathrm{df}=6,36, \mathrm{GG}=0.001)$ at all points except baseline (Figure 3). Even though the TRP/LNAA ratio decreased with the $1 / 4$ strength drink from 0.105 at baseline to 0.056 and 0.062 at 17:00 and 19:00, respectively, these changes were not significant $(G G=0.062)$.

The nadir of TRP at 15:00 with the $1 / 4$ drink was greater than with the original control drink administered at 08:00 (Delgado et al. 1990, 1994). In this regard it should be recalled that TRP levels in the natural state have a circadian rhythm with the lowest point at 08:00 and the peak at 24:00 to 3:00 (Steardo et al. 1980; Wurtman et al. 1968).

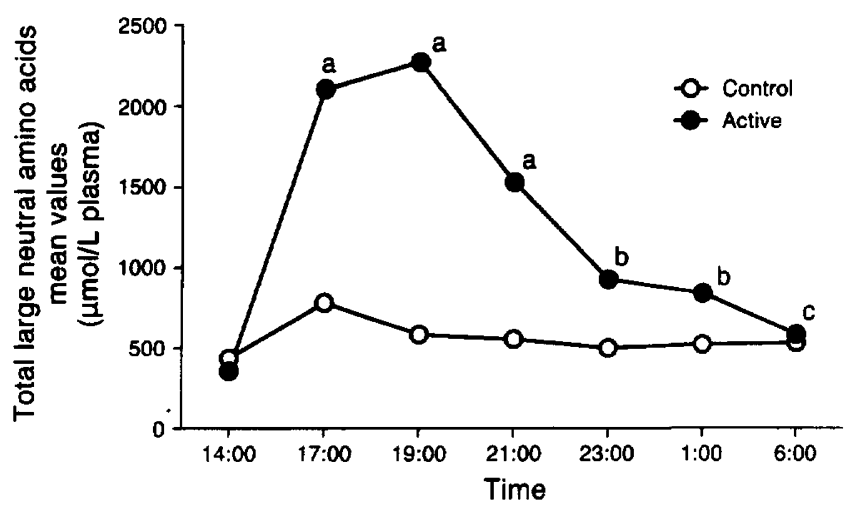

Figure 2. Mean total large neutral amino acids. Significant difference between active and control $(n=7), a, p<.004 ; b, p<$ $.0068 ; c, p<.01$. 


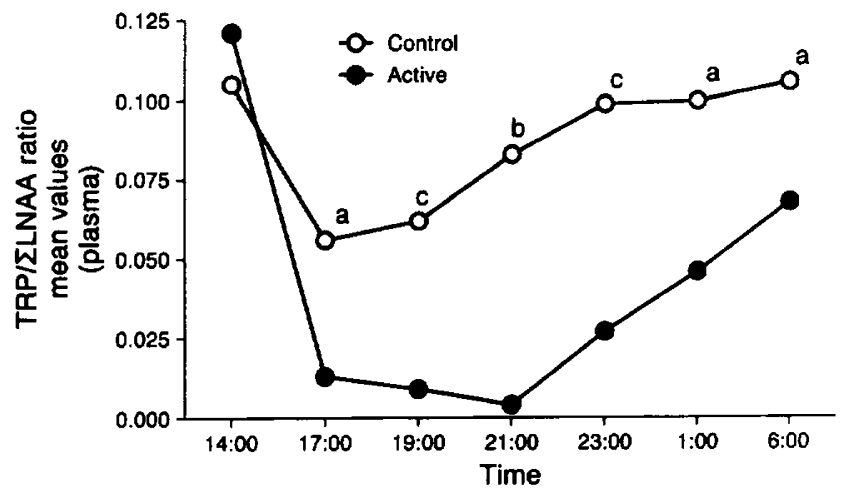

Figure 3. Ratio of mean tryptophan over mean total large amino acids. Significant difference between active and control $(n=7), a, p<.02 ; b, p<.0001 ; c, p<.005$.

Nocturnal urinary 6-MS decreased significantly from $5.98 \mu \mathrm{g} / 8$ hours to $3.39 \mu \mathrm{g} / 8$ Hours for the full-strength versus control drink $(F=8.37, \mathrm{df}=2,12, \mathrm{GG}=0.024$ : Figure 4).

\section{DISCUSSION}

This study demonstrated that administering a control drink consisting of an AAM of $1 / 4$ strength of the preparation currently used to induce TRP depletion lowered total plasma TRP levels slightly from a baseline value of $44.1 \mu \mathrm{mol} / \mathrm{ml}$ to about $35 \mu \mathrm{mol} / \mathrm{ml}$ at the first two blood draws with return to baseline 2 hours later (Delgado et al. 1990). During the active experiment (100 mg of AAM), total plasma TRP levels decreased to a minimum value of $6 \mu \mathrm{mol} / \mathrm{ml}$ after 7 hours. Overall the degree of change in mean total TRP was small in the control versus active experiment. Nonetheless the slight reduction in plasma TRP levels indicated that the protein synthesis initiated with the $1 / 4$ strength drink in-

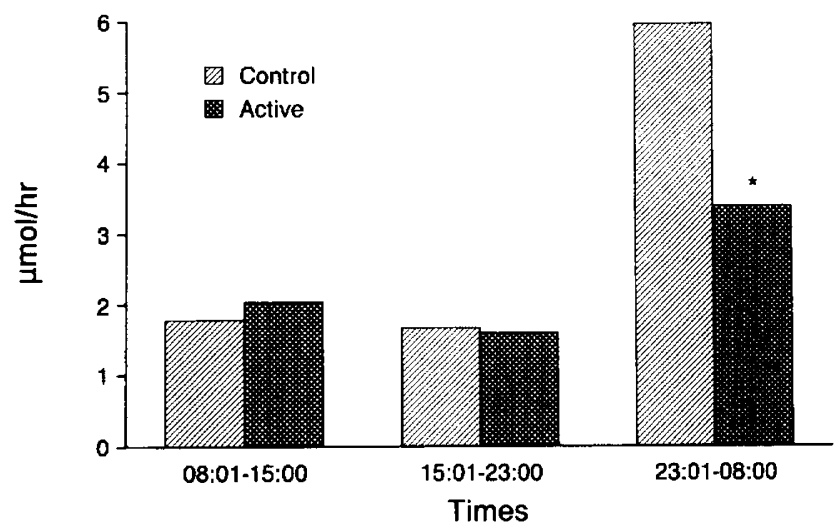

Figure 4. Urinary 6-hydroxymelatonin sulfate. Significant difference between active and control $(n=7), a, p<.024$. corporated a significant, albeit small, amount of TRP for a limited time (5 hours).

Changes induced by TRP depletion were superimposed on the endogenous cycle of TRP. These conditions may explain the observations of lower TRP levels with the 1/4 strength control drink administered in the morning compared to the early afternoon. This may also clarify why signs and symptoms of depression were noted previously when the $1 / 4$ strength drink was given in the morning to vulnerable subjects (Moreno et al. 1995). In contrast to the control drinks, the TRP levels between the modified and original techniques were similar with the full-strength preparation (Delgado et al. 1990). As Figure 1 indicates, the active drink was potent enough to deplete TRP effectively, even with the contribution of the TRP diurnal rhythm.

Given the relatively small sample size of seven subjects, the statistically insignificant change in the TRP/ LNAA ratio may represent a type II error. Nonetheless, the observation that the TRP/LNAA ratio is slightly lower in the control condition at the first two blood draws before returning to baseline at the 7-hour mark merits examination. This change is consistent with the interpretation of the TRP diurnal rhythm discussed previously. In effect the TRP/LNAA ratio defines the amount of TRP that enters the brain (Weltzin et al. 1994). In contrast, during the active experiment, the ratio is 25 times lower than baseline 2 hours after administration and remains low for another 8 hours. Previous studies used a 100-mg AAM with $2.3 \mathrm{~g}$ of TRP (Delgado et al. 1990, 1994). The dose of TRP was intended to keep the TRP/ LNAA ratio close to the baseline ratio. Recently, it was shown that such a mixture significantly reduced the plasma TRP/LNAA ratio to about $45 \%$ of baseline for at least 7 hours after the drink was ingested (Weltzin et al. 1994). Therefore, the TRP/LNAA ratios induced with the $1 / 4$ preparation are very similar to those reported using Delgado's technique (Delgado et al. 1994).

The concentration of LNAA increased slightly with the $1 / 4$ drink, from $43.9 \mu \mathrm{mol} / \mathrm{ml}$ to $78.3 \mu \mathrm{mol} / \mathrm{ml} 3$ hours after the drink and then fluctuated around 50.0 $\mu \mathrm{mol} / \mathrm{ml}$. In contrast, during the administration of the active drink the concentration of LNAA increased to over $2,000 \mu \mathrm{mol} / \mathrm{ml}$ and returned to baseline only 15 hours later. These data indicated that the LNAA that competes with TRP for transport across the blood-brain barrier might inhibit TRP transport slightly during the control condition.

Subjects were unable to distinguish between the two preparations. In general they reported mild to moderate nausea with both drinks. Their inability to discern differences in taste, texture, or side effects was likely enhanced by the 4 weeks between the two study phases. This time interval was selected to limit the study to the early follicular phase of the menstrual cycle and ensure normalization of the relevant circadian rhythms. 
Consistent with earlier studies (Zimmermann et al. 1993), the urinary metabolite of melatonin 6-MS decreased significantly during the active versus control. This finding indicated that the synthesis of melatonin and 6-MS, whose production depends on TRP and serotonin, was less affected by the $1 / 4$ strength drink. To clarify whether the $1 / 4$ drink indeed acts a true control, it would be necessary to repeat the study with an inactive control.

In summary this study demonstrated that changes in TRP, LNAA, TRP/LNAA ratio, and 6-MS during the active experiment were consistent with the original technique. More important, compared with the original, the modified control drink administered at 15:00 hours induced only a minor decrease in TRP, a slight increase in LNAA, and less change in the TRP/LNAA ratio. Finally the $1 / 4$ strength drink did not alter the stable metabolite of indoleamine synthesis 6-MS. This observation is consistent with findings from a previous study with a similar control drink (Zimmermann et al. 1993). Because subjects reported that they could not differentiate between the two drinks, this modified procedure permits rapid TRP depletion in double-blind, placebocontrolled studies without using TRP. Study participants are therefore not at risk for eosinophilia myalgia syndrome. Studies using this modified technique to study vulnerable subjects are in progress.

\section{ACKNOWLEDGMENTS}

This project was supported by a NARSAD Young Investigator Award to RCZ and the General Clinical Research Center (GCRC), Mayo Foundation (grant \# M01 RR585). The authors wish to thank the nursing staff of the GCRC, particularly study coordinator Jean Feehan-Hofbauer, R.N. We also appreciate the work of Ms. Darlene Lucas (6-MS assays) and James D. Jones, Ph.D. (amino acids analysis).

\section{REFERENCES}

Delgado PR, Charney DS, Price LH, Aghajanian GK, Landis H, Heninger GR (1990): Serotonin function and the mechanism of antidepressant action. Arch Gen Psychiatry 47:411-418

Delgado PR, Price LH, Miller HL, Salomon AM, Aghajanian GK, Heninger GR, Charney DS (1994): Serotonin and the neurobiology of depression: Effects of tryptophan depletion in drug-free depressed patients. Arch Gen Psychiatry 51:865-874

Centers for Disease Control and Prevention (1991): Eosinophilia-myalgia syndrome: Follow-up survey of patients, New York 1990-1991. MMWR 40:401-403

Lucca A, Lucini V, Piatti E, Ronchi P, Smeraldi E (1992): Plasma tryptophan levels and plasma tryptophan/large neural amino acids in patients with mood disorder, patients with obsessive-compulsive disorder and normal subjects. Psychiatry Res 44:85-91

Moreno FA, Panov S, Strayer LJ, Potter RL, Gelenberg AJ, Delgado PL (May 1995): Tryptophan Depletion and Vulnerability to Depression. New Research Abstract No. NR97, presented at the American Psychiatric Association Annual Meeting.

Rosse RB, Schwartz BL, Zlotolow S, Banay-Schwartz M, Trinidad AC, Peace TD, Deutsch SI (1992): Effect of a low-tryptophan diet as an adjuvant to conventional therapy in schizophrenia. Clin Neuropharmacol 15(2): 129-141

Steardo L, Bonuso S, Pisanti N, Marano E (1980): Circadian rhythm of tryptophan in normal volunteers. Acta Neurologica 2(2):86-93

Weltzin TE, Fernstrom JD, McConaha C, Kaye WH (1994): Acute tryptophan depletion in bulimia: Effects on large neutral amino acids. Biol Psychiatry 35(6):388-397

Wurtman RJ, Rose CM, Chou C, Larin FF (1968): Daily rhythms in the concentration of various amino acids in human plasma. N Eng J Med 279:171-175

Zimmermann RC, McDougle CJ, Schumacher M, Olcese J, Heninger GR, Price LH (1993): Urinary 6-Hydroxymelatonin sulfate as a measure of melatonin secretion during acute tryptophan depletion. Psychoneuroendocrinology $18: 567-578$ 